\title{
A!
}

This is an electronic reprint of the original article.

This reprint may differ from the original in pagination and typographic detail.

Räsänen, Esa; Harju, Ari; Puska, Martti; Nieminen, Risto M.

\section{Rectangular quantum dots in high magnetic fields}

Published in:

Physical Review B

DOI:

10.1103/PhysRevB.69.165309

Published: 14/04/2004

Document Version

Publisher's PDF, also known as Version of record

Please cite the original version:

Räsänen, E., Harju, A., Puska, M., \& Nieminen, R. M. (2004). Rectangular quantum dots in high magnetic fields.

Physical Review B, 69(16), 1-5. [165309]. https://doi.org/10.1103/PhysRevB.69.165309

This material is protected by copyright and other intellectual property rights, and duplication or sale of all or part of any of the repository collections is not permitted, except that material may be duplicated by you for your research use or educational purposes in electronic or print form. You must obtain permission for any other use. Electronic or print copies may not be offered, whether for sale or otherwise to anyone who is not an authorised user. 


\title{
Rectangular quantum dots in high magnetic fields
}

\author{
E. Räsänen, * A. Harju, M. J. Puska, and R. M. Nieminen \\ Laboratory of Physics, Helsinki University of Technology, P.O. Box 1100, FIN-02015 HUT, Finland
}

(Received 27 November 2003; revised manuscript received 29 January 2004; published 14 April 2004)

\begin{abstract}
We use density-functional methods to study the effects of an external magnetic field on two-dimensional quantum dots with a rectangular hard-wall confining potential. The increasing magnetic field leads to spin polarization and formation of a highly inhomogeneous maximum-density droplet at the predicted magneticfield strength. At higher fields, we find an oscillating behavior in the electron density and in the magnetization of the dot. We identify a rich variety of phenomena behind the periodicity and analyze the complicated many-electron dynamics, which is shown to be highly dependent on the shape of the quantum dot.
\end{abstract}

DOI: 10.1103/PhysRevB.69.165309

PACS number(s): 73.21.La, 71.10.-w

\section{INTRODUCTION}

The problem of many interacting electrons in a noncircular quantum well under the influence of an external magnetic field is extremely challenging for the computational tools available. For the time being, theoretical studies of such systems have dealt either with very small electron numbers, or with the single-electron properties in nonintegrable quantum billiards. ${ }^{1}$ On the other hand, the rapid technical development in the realization of different quantum-dot systems naturally motivates theoretical modeling of complicated many-electron structures. ${ }^{2}$

The magnetic-field dependence of the energy spectrum in a square two-electron quantum dot has been analyzed in detail by Creffield et al. ${ }^{3}$ They found Aharonov-Bohm-type oscillations in the lowest levels, indicating periodic singlettriplet changes in the ground state. Ugajin ${ }^{4}$ found that these state transitions lead to strong effects in the optical excitation spectra. Within a similar square-dot system, he studied the effects of the Coulomb interaction on the far-infraredabsorption (FIR) spectra. ${ }^{5}$ Recent density-functional FIR calculations for soft-wall triangular and square dots have been done by Valín-Rodríquez et al., ${ }^{6}$ who identified corner and side modes in the system. The interactions and the quantumdot geometry affect also strongly the magnetization, at least for small electron numbers. ${ }^{7,8}$

In a circular geometry, a maximum-density droplet (MDD) caused by the magnetic field corresponds to a polarized state with electrons occupying successive angular momentum states, i.e., $l=0,-1, \ldots,-N+1$, giving $L_{\mathrm{MDD}}$ $=-\frac{1}{2} N(N-1)$ for the total angular momentum of $N$ electrons. A stability region for the MDD has been identified in electron-transport experiments through vertical quantum dots by Oosterkamp et al. ${ }^{9}$ This state, as well as the post-MDD regime in higher magnetic fields, has been considered theoretically in circular parabolically confined quantum dots by several authors. ${ }^{10-15}$ In the case of a noncircular symmetry, however, the high-magnetic-field limit has not been previously analyzed to the best of our knowledge.

In this work, we examine the magnetic-field effects in rectangular quantum dots by using the spin-densityfunctional theory (SDFT). Our aim is to define the MDD state in a rectangular geometry and extend our previous analysis of the MDD formation in hard-wall quantum dots. ${ }^{16}$
The onset of the MDD can be predicted from the number of flux quanta which only depends on the area of the dot and is thus geometry independent. We study also the beyond-MDD regime that can be characterized by periodic state oscillations. The periodicity can be observed in the magnetization which we compare with the corresponding results for noninteracting electron systems. The origin of the periodicity is generally the competition between magnetic confinement and Coulomb repulsion, but the behavior of the effective single-electron states is very sensitive to the dot geometry.

This paper is organized as follows. In Sec. II we present briefly the model Hamiltonian and the computational methods, based on a real-space spin-density-functional approach. In Sec. III we consider the formation and the structure of the MDD in rectangular geometries. Section IV presents the state oscillations above the MDD regime, first from the point of view of the total magnetization, and finally at the level of the effective single-electron states. The paper is summarized in Sec. V.

\section{THE MODEL AND THE METHODS}

We restrict the dot to the $x y$ plane and use the effectivemass approximation with the material parameters for GaAs, i.e., the effective mass $m^{*}=0.067 m_{e}$ and the dielectric constant $\epsilon=12.7$. The many-body Hamiltonian reads

$$
\begin{aligned}
H= & \frac{1}{2 m^{*}} \sum_{i=1}^{N}\left[-i \hbar \nabla_{i}+e \mathbf{A}\left(\mathbf{r}_{i}\right)\right]^{2}+\sum_{i<j}^{N} \frac{e^{2}}{4 \pi \epsilon_{0} \epsilon\left|\mathbf{r}_{i}-\mathbf{r}_{j}\right|} \\
& +\sum_{i=1}^{N}\left[V_{\mathrm{ext}}\left(\mathbf{r}_{i}\right)+g^{*} \mu_{B} B s_{z, i}\right],
\end{aligned}
$$

where the shape of the dot is defined by a rectangular hardwall confining potential,

$$
V_{\text {ext }}(x, y)= \begin{cases}0, & 0 \leqslant x \leqslant \beta L, 0 \leqslant y \leqslant L \\ \infty & \text { otherwise }\end{cases}
$$

Here $\beta$ defines the ratio of the side lengths of the rectangle, and $L$ is scaled such that the area of the dot remains constant, i.e., $\beta L^{2}=4 \pi^{2} a_{B}^{* 2}$, where $1 a_{B}^{*} \approx 10.03 \mathrm{~nm}$. We apply an external magnetic field $\mathbf{B}=B \hat{z}$ perpendicular on the $x y$ plane, and use the symmetric gauge, 

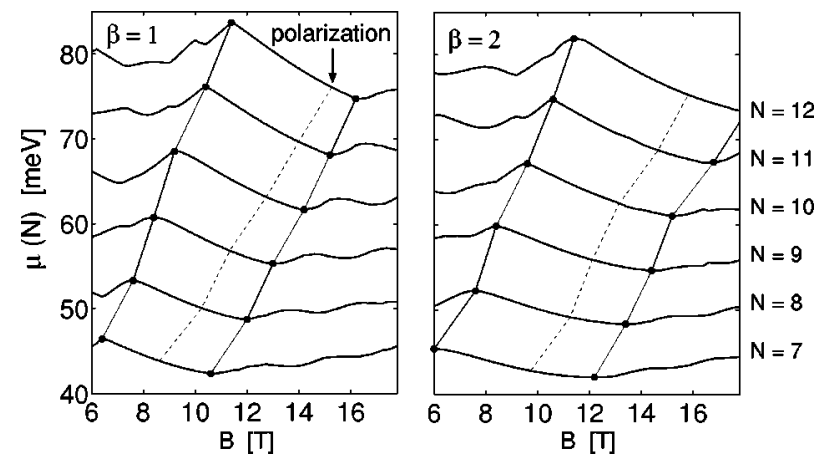

FIG. 1. Chemical potentials of the fully polarized states for $N$-electron rectangular quantum dots as a function of the magnetic field $B$.

$$
\mathbf{A}=\frac{B}{2}(-y, x, 0),
$$

for the vector potential. The last term in Eq. (1) is the Zeeman energy (with $g^{*}=-0.44$ for GaAs) which has an insignificant effect on the results presented and is thus neglected. In the calculations we employ the SDFT in the selfconsistent Kohn-Sham (KS) formulation. We tested that in the systems considered in this study, the SDFT gives reasonably accurate results compared to the computationally more demanding current-spin-density-functional approach. ${ }^{2,17}$ In the local-spin-density approximation we use the exchangecorrelation energy by Attaccalite et al. ${ }^{18}$ It is based on the diffusion Monte Carlo simulations over the whole range of spin polarization, which is a major improvement compared to the previous parametrizations. This leads to more accurate results in both zero and nonzero magnetic fields as shown in Ref. 19.

The calculations are performed in real space using finite differences for the derivative operations on two-dimensional point grids. Since there are no implicit restrictions for the symmetry, the external potential can be shaped arbitrarily in the computing region. The number of grid points is 128 $\times 128$, giving a sufficient accuracy in the total energy. To make the convergence effective, we employ the Rayleigh quotient multigrid method ${ }^{20}$ for the discretized singleelectron Schrödinger equations.

\section{MDD FORMATION}

In the zero-field solution for a rectangular quantum dot with an even number of electrons, the total spin is either 0 or 1 , depending on the degeneracy on the highest level. ${ }^{21}$ Increasing $B$ brings the higher spin states closer to the $S=0$ and $S=1$ states in energy, and finally the full spin polarization is found. The value of $B$ for the complete spin polarization is rather independent of the deformation parameter. ${ }^{16}$ In the eight-electron dot, for example, the ground state becomes fully spin polarized at $B \sim 10 \mathrm{~T}$ as $\beta=1$ and at $11 \mathrm{~T}$ as $\beta=2$.

Figure 1 shows the chemical potentials $\mu(N)=E(N)$ $-E(N-1)$ of the polarized states as a function of $B$ for $N$ $=7-12$. The point when the fully polarized state becomes

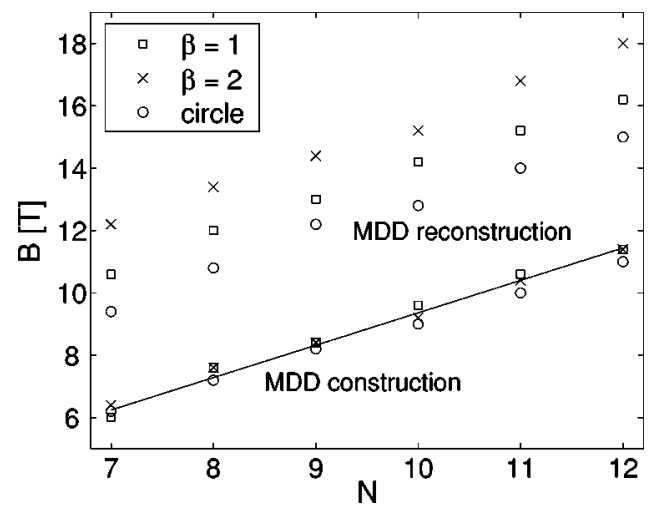

FIG. 2. MDD-window limits as a function of the number of electrons in different quantum-dot geometries. The line for the predicted MDD formation is also shown.

the ground state is marked with a dotted line in the figure. We consider only the polarized state in order to clarify the MDD window, which corresponds to the descending regime in the chemical potential. Similar behavior in $\mu$ was observed in the experiments for vertical quantum dots by Oosterkamp et al. ${ }^{9}$ They measured the evolution of the Coulomb blockade peaks as a function of $B$ for $N=0-40$ and found clearly identifiable phases for the filling factor, of which $\nu=1$ corresponds to a maximum-density droplet. According to our definition, the true MDD exists on the rightmost ascending stripe in Fig. 1. As $\beta$ increases, the MDD windows become larger and flatter due to pronounced localization in the $x$ direction. Thus, the square case shows the most drastic behavior because of the highest consistency of the dot symmetry with the magnetic confinement.

Figure 2 gives the limits for the MDD window as a function of $N$ in different quantum-dot geometries. We see that the increase in the width of the MDD window is due to a shift in the reconstruction point, whereas the onset of the window is nearly independent of the dot geometry. The reason is the following. The formation of the MDD requires an equal number of flux quanta, $N_{\Phi}=\Phi / \Phi_{0}$, to the $N-1$ vortices "seen" by an electron as the other electrons are fixed. A vortex is defined as a point where the wave function is zero and its phase changes by $-2 \pi$ for a counterclockwise rotation. ${ }^{22}$ Here $\Phi_{0}=h / e$ is the single-flux quantum, and since the magnetic flux is directly proportional to the dot area, i.e., $\Phi=B A$, we thus get $B=h(N-1) / e A \approx 1.04(N$ $-1) \mathrm{T}$ for the onset of the MDD in all geometries. We show this prediction in Fig. 2 and it agrees remarkably well with the transition points obtained from the chemical potentials. We remark that the electron-electron interactions are a prerequisite for this agreement, so the pure single-electron picture would be insufficient for the prediction presented above. The reconstruction limit is, however, much more difficult to estimate and it depends strongly on the geometry as explained above.

At the end of the MDD window the droplet achieves the smoothest electron density. In Fig. 3 we show the maximumdensity distributions for $\beta=1$ and $\beta=2$ quantum dots, and also for a circular hard-wall dot with $N=8$. They have a highly inhomogeneous structure instead of a flat distribution 


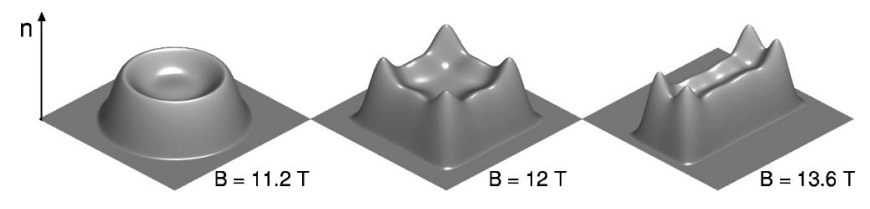

FIG. 3. Electron densities for the MDD states of eight-electron quantum dots with different geometries.

found in parabolically confined MDD's. ${ }^{13}$ This is a direct consequence of the hard-wall confinement where the Coulomb interaction favors strong localization in the corners and on the edges over the whole range of $B$. In the circular hardwall MDD, the occupation of the angular momentum orbitals follows the original definition of a maximum-density droplet for a parabolic dot, i.e., $l_{z}=0,-1, \ldots,-7$. The number of vortices in a particular KS state thus equals $\left|l_{z}\right|$. For $N$ electrons at the positions $z_{i}=x_{i}+i y_{i}$, the many-body wave function for a parabolic MDD can be written in Laughlin's ${ }^{23}$ form as

$$
\Psi_{\mathrm{MDD}}=\exp \left(-\frac{\pi}{2} B \Phi_{0} \sum_{k}\left|z_{k}\right|^{2}\right) \prod_{i<j}^{N}\left(z_{i}-z_{j}\right),
$$

which is similar to the wave function for a state with the filling factor $\nu=1$ in the thermodynamic limit. It is easy to see that by following the above definition for a vortex we obtain the correct total number of $N-1$ vortices in the system.

The rectangular dot shows a more complicated structure: the formation of the MDD corresponds to the appearance of the correct maximum number of $N-1$ vortices in the KS state highest in energy, but in the other states there are typically "extra" vortices, leading to more than $N(N-1) / 2$ of them in the KS states altogether. The reason is the complex structure of the indefinite angular momentum states that prevents the description of the noncircular MDD with a Laughlin-type many-body wave function, even if the $B-N$ dependence for the MDD formation can be correctly estimated. Thus, we resort to considering the evolution of the expectation values of the angular momentum operator, $\hat{l}_{z}=-i \hbar[x(\partial / \partial y)-y(\partial / \partial x)]$, for different KS states in the high- $B$ regime. Below we designate this expectation value for noncircular dots as the effective $l_{z}$.

\section{STATE OSCILLATIONS}

The rapidly growing Coulomb interaction leads to the reconstruction of the MDD into a lower-density droplet with a higher angular momentum as $B$ increases. According to our calculations, the collapse of the circular hard-wall MDD leads into a ringlike structure with $l_{z}=-1,-2, \ldots,-N$, corresponding to the creation of a hole in the center. For small parabolic dots, a similar symmetry-preserving structure has been obtained with the exact diagonalization by Yang and MacDonald. ${ }^{12}$ Especially for large parabolic systems, there are also clear signs of edge reconstruction of the MDD in the form of strong charge-density waves, ${ }^{13}$ Wigner crystalline edges, ${ }^{14}$ or spin texturing. ${ }^{15}$ In our rectangular hardwall quantum dot, the MDD collapses into a strongly local-

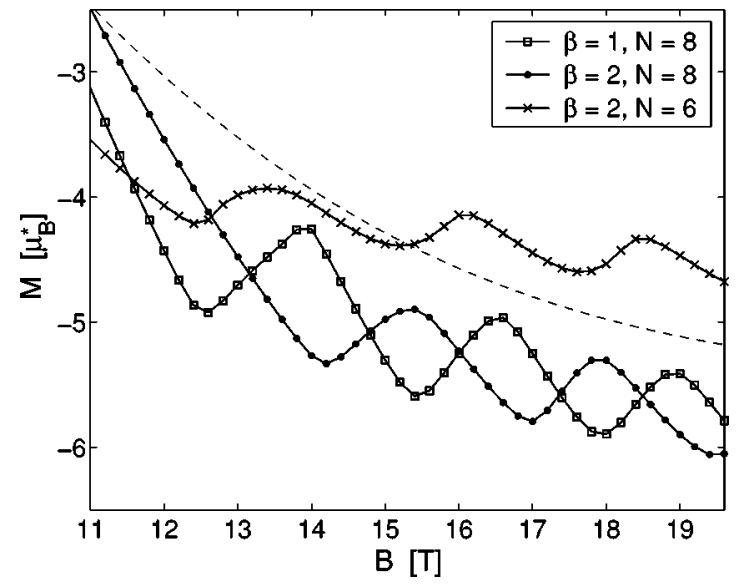

FIG. 4. Magnetization in three different rectangular quantumdot systems beyond the MDD regime. The dashed line indicates the noninteracting case of $(\beta, N)=(2,6)$. The effective Bohr magneton $\mu_{B}^{*}=e \hbar / 2 m^{*}$.

ized edge state that resembles a Wigner molecule in the sense that there are pronounced peaks in the electron density to minimize the Coulomb interaction. ${ }^{24}$

As $B$ is increased further, the electronic structure begins to oscillate between well-localized and more diffuse states. This is a result from the interplay between the magnetic confinement and the Coulomb interaction. In this connection, the fully polarized ground state changes periodically as the interactions entangle the single-electron states on the lowest Landau level. This is similar to singlet-triplet oscillations found in interacting two-electron quantum dots of both circular $^{25}$ and square ${ }^{3}$ shapes, as well as in a double-dot system. ${ }^{26}$ We emphasize, however, that in our case the ground state remains fully polarized beyond the MDD, i.e., $S_{z}=N / 2$.

\section{A. Magnetization}

The structural oscillations beyond the MDD state can be observed in the magnetization of the dot, since the growth in the total angular momentum leads to the decrease in the current. The total magnetization for a finite system is defined at zero temperature as

$$
M=-\frac{\partial E_{\text {tot }}}{\partial B} .
$$

In Fig. 4 we show the magnetization in three rectangular quantum dots beyond the MDD regime. The local minima correspond to the points where the localization on the edge is maximized, and at local maxima there are states with a density contribution at the center. It can be clearly seen in Fig. 4 that $M$ decreases with increasing electron number, and the onset of the oscillation moves to higher $B$ as the deformation $\beta$ is increased, which is consistent with the upper limit of the MDD window shown in Fig. 2. The period in magnetic-field strength is nearly a constant, $\sim 2.5 \mathrm{~T}$ in all the systems presented in Fig. 4. We emphasize that the majority of $(\beta, N)$ combinations lead to more complicated and even irregular magnetization. This will be discussed in more detail in Sec. 


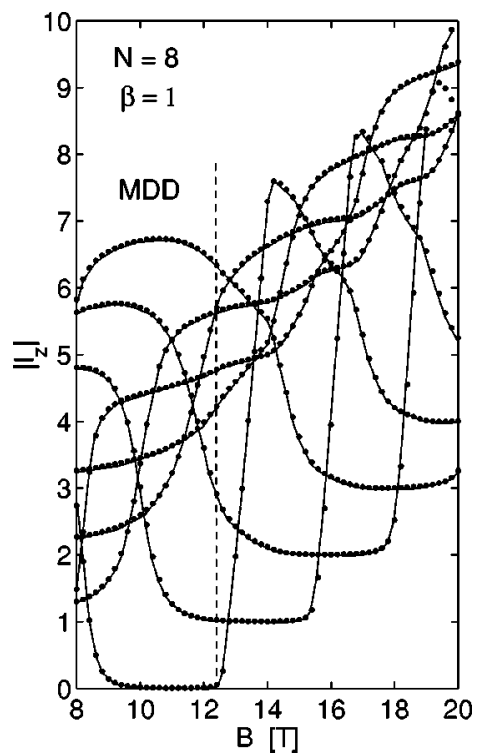

FIG. 5. Evolution of the effective $l_{z}$ values as a function of the magnetic field $B$ in a square eight-electron quantum dot.

IV B. Figure 4 also shows the noninteracting magnetization for $(\beta, N)=(2,6)$, calculated from the six lowest eigenenergies on the first Landau level. In the MDD regime, it is $\sim 1 \mu_{B}^{*}$ above the interacting curve with the same slope. At $B \sim 15 \mathrm{~T}$, however, there is a clear crossing which indicates that the interactions retard the overall increase in $|M|$ as a function on $B$ in the oscillatory regime. As can be seen in Fig. 4, the retardation is due to the decreasing parts in $|M|$. The first of them correspond to the reconstruction of the MDD when the Coulomb energy collapses stronger than the kinetic energy increases. The same occurs periodically at higher magnetic fields, leading to lower $|M|$ than in the single-electron picture.

Magnetization in square-shaped quantum dots up to $N$ $=4$ has been studied by Magnúsdóttir and Gudmundsson. ${ }^{7}$ They found remarkably higher magnetization in the noninteracting than in the interacting picture, but did not consider the high- $B$ limit, where we see the crossing between those two cases. In a two-electron square dot studied by Sheng and $\mathrm{Xu}^{8}{ }^{8}$ the magnetization looks similar to our case, but the oscillation represents the trivial singlet-triplet exchange.

\section{B. Development of the angular momenta}

As a mean-field theory, the density-functional approach only approximates the many-body energy states. However, we will next consider the development of the effective angular momentum of different $\mathrm{KS}$ orbitals in order to obtain a deeper insight on the state oscillations on the single-electron level. In Fig. 5 we present the evolution of the effective $l_{z}$ values of the occupied KS levels in the polarized state of a square eight-electron quantum dot as a function of $B$. The structure is intriguing and shows clearly two different modes in the system. First, there are four closely entangled states, representing the relatively stable corner modes. They are reminiscent of the Aharonov-Bohm-like energy-level structure in an interacting two-electron dot. ${ }^{3}$ Second, there are

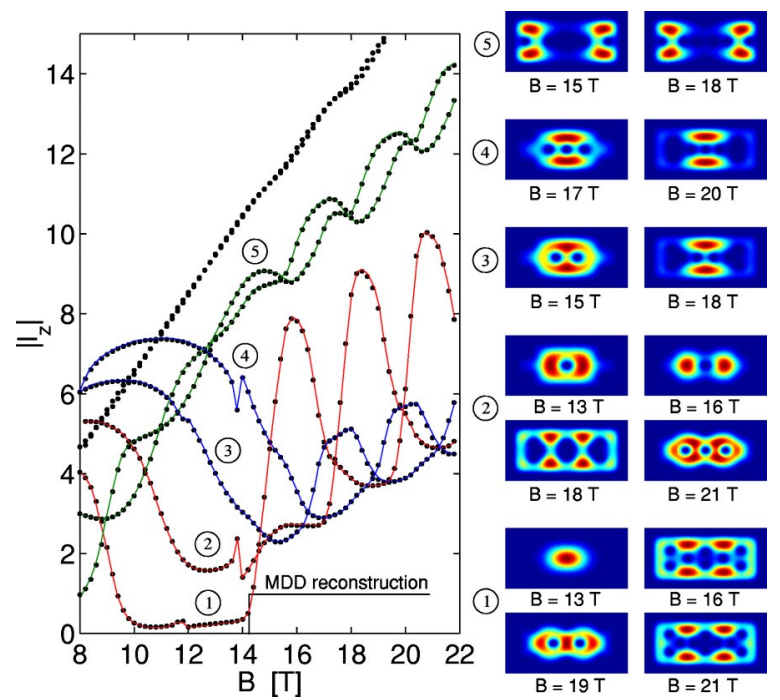

FIG. 6. (Color online) Evolution of the effective $l_{z}$ values as a function of the magnetic field $B$ in a $\beta=2$ eight-electron quantum dot. KS wave functions, corresponding to different modes, are also shown.

states that with increasing $B$ condense to nearly circular orbits on the first Landau level before the Coulomb interaction expands them to high effective $l_{z}$ values. In this context, the state development is similar to the circular case, where the occupation shifts from the lowest $l_{z}$ state to the highest level as the ground state changes. The periodic spread of the Landau states is clearly the origin of the magnetization oscillations discussed above. The upper limit of the MDD window, marked in Fig. 5, can be interpreted as the most evenly extended effective $l_{z}$ distribution similar to the corresponding circularly symmetric system.

Figure 6 shows the development of the effective $l_{z}$ values in the case of $\beta=2$. Due to the reduction of the symmetry, the evolution is more complicated than in the square case, and we can find four different modes. First, there are two corner modes in the system. Of them, the highest effective $l_{z}$ state shows steady localization at the both ends of the rectangle, and the second one (5) oscillates weakly. The latter is visualized in the right panel of Fig. 6 with the KS wave functions, $\left|\psi_{\mathrm{KS}}^{i}\right|^{2}$ (see also Ref. 27). In addition, there are two different types of highly oscillatory states. The first pair $(3,4)$ has a remarkably lower amplitude in the oscillation regime $(B \geqslant 14 \mathrm{~T})$. As can be seen in the $\mathrm{KS}$ wave functions, they correspond to localization in the $y$ direction. Interlocked to these states, there are strongly oscillative states $(1,2)$ that, according to the $\mathrm{KS}$ wave functions, seem to form a periodic structure in the rectangle, reminiscent of a "lozenge" orbit in the corresponding classical bouncing map, studied in the square case by Aguiar. ${ }^{28}$ It is noticeable that these states affect strongly on the corner modes by compressing them slightly to lower $l_{z}$. The cusps at $B=11.8 \mathrm{~T}$ and $13.8 \mathrm{~T}$ are numerical noise, corresponding to degeneracies of the KS orbitals 1 and 3 or 2 and 4, respectively.

It is clear from Fig. 6 that the formation of bulk Landau states disappears as the dot is squeezed toward a rectangular shape. Thus, the electron-electron interactions make the 
quantum dot sensitive to the deformation parameter. On the other hand, the overall behavior beyond the MDD is qualitatively similar in all geometries, even if the evolution of the effective $l_{z}$ values gets very complicated. This occurs, e.g., in the case of $\beta=1.5$, where the different modes discussed above are hardly recognizable. In this sense, an increase in the symmetry separates the different states and makes the corresponding classically stable orbits visible.

We underline that the KS orbitals given by the densityfunctional theories do not have a direct physical interpretation. In this particular system, however, we find that the procedure gives important information on the many-particle dynamics in an interacting nonintegrable quantum dot, not accessible with more "exact" computational methods.

\section{SUMMARY}

To summarize, we have studied the electronic structure of rectangular quantum dots in a magnetic field. We have identified the formation of a maximum-density droplet at the predicted $B$, even if the electron density is strongly localized due to the hard-wall confinement. Beyond the MDD, we have found oscillating magnetization that reflects the peri-

*Electronic address: ehr@fyslab.hut.fi

${ }^{1}$ H.-J. Stockmann, Quantum Chaos: An Introduction (Cambridge University Press, Cambridge, 2000).

${ }^{2}$ For a review of quantum dots, see S.M. Reimann and M. Manninen, Rev. Mod. Phys. 74, 1283 (2002).

${ }^{3}$ C.E. Creffield, J.H. Jefferson, S. Sarkar, and D.L.J. Tipton, Phys. Rev. B 62, 7249 (2000).

${ }^{4}$ R. Ugajin, Phys. Rev. B 53, 6963 (1996).

${ }^{5}$ R. Ugajin, Phys. Rev. B 51, 10714 (1995).

${ }^{6}$ M. Valín-Rodríquez, A. Puente, and L. Serra, Phys. Rev. B 64, 205307 (2001)

${ }^{7}$ I. Magnúsdóttir and V. Gudmundsson, Phys. Rev. B 61, 10229 (2000).

${ }^{8}$ W. Sheng and H. Xu, Physica B 256-258, 152 (1998).

${ }^{9}$ T.H. Oosterkamp, J.W. Janssen, L.P. Kouwenhoven, D.G. Austing, T. Honda, and S. Tarucha, Phys. Rev. Lett. 82, 2931 (1999).

${ }^{10}$ M. Ferconi and G. Vignale, Phys. Rev. B 56, 12108 (1997).

${ }^{11}$ A. Harju, S. Siljamäki, and R.M. Nieminen, Phys. Rev. B 60, 1807 (1999).

${ }^{12}$ S.-R. Eric Yang and A.H. MacDonald, Phys. Rev. B 66, 041304(R) (2002).

${ }^{13}$ S.M. Reimann, M. Koskinen, M. Manninen, and B.R. Mottelson, Phys. Rev. Lett. 83, 3270 (1999).

${ }^{14}$ E. Goldmann and S.R. Renn, Phys. Rev. B 60, 16611 (1999). odic changes in the polarized ground state. In the oscillating regime, the magnetization shifts to lower values than in the corresponding noninteracting case. The origin of the periodic behavior has been analyzed by following the evolution of the effective angular momentum quantum numbers. There are clear corner modes in all rectangular systems, but the development of the center orbits is found to be very sensitive to the geometry of the dot. In a square, the central KS orbitals are nearly symmetric, whereas a rectangle with $\beta=2$ shows two different oscillative modes. When the symmetry is reduced further, the center orbitals become unindentifiable. In spite of the limitations in the mean-field approach, we have managed to bring out the richness of phenomena exhibited by the electronic structure in the high- $B$ limit. We hope that this would inspire new experimental work on this topic.

\section{ACKNOWLEDGMENTS}

This research has been supported by the Academy of Finland through its Centers of Excellence Program (2000-2005). E.R. is also grateful to Magnus Ehrnrooth foundation for financial support.

${ }^{15}$ A. Karlhede, S.A. Kivelson, K. Lejnell, and S.L. Sondhi, Phys. Rev. Lett. 77, 2061 (1996).

${ }^{16}$ E. Räsänen, M.J. Puska, and R.M. Nieminen, Physica E (Amsterdam) 22, 490 (2004).

${ }^{17}$ G. Vignale and M. Rasolt, Phys. Rev. Lett. 59, 2360 (1987).

${ }^{18}$ C. Attaccalite, S. Moroni, P. Gori-Giorgi, and G.B. Bachelet, Phys. Rev. Lett. 88, 256601 (2002); 91, 109902(E) (2003).

${ }^{19}$ H. Saarikoski, E. Räsänen, S. Siljamäki, A. Harju, M.J. Puska, and R.M. Nieminen, Phys. Rev. B 67, 205327 (2003).

${ }^{20}$ M. Heiskanen, T. Torsti, M.J. Puska, and R.M. Nieminen, Phys. Rev. B 63, 245106 (2001).

${ }^{21}$ E. Räsänen, H. Saarikoski, V.N. Stavrou, A. Harju, M.J. Puska, and R.M. Nieminen, Phys. Rev. B 67, 235307 (2003).

${ }^{22}$ T. Chakraborty and P. Pietiläinen, The Quantum Hall Effects: Fractional and Integral (Springer, Berlin, 1995).

${ }^{23}$ R.B. Laughlin, Phys. Rev. Lett. 50, 1395 (1983).

${ }^{24}$ E. Räsänen, H. Saarikoski, M.J. Puska, and R.M. Nieminen, Phys. Rev. B 67, 035326 (2003).

${ }^{25}$ M. Wagner, U. Merkt, and A.V. Chaplik, Phys. Rev. B 45, 1951 (1992).

${ }^{26}$ A. Harju, S. Siljamäki, and R.M. Nieminen, Phys. Rev. Lett. 88, 226804 (2002).

${ }^{27}$ Animations of the KS states can be found from URL http:// www.fyslab.hut.fi/arch/ehr/recta.html

${ }^{28}$ M.A.M. de Aguiar, Phys. Rev. E 53, 4555 (1996). 